\title{
The lung ultrasound: facts or artifacts? In the era of COVID-19 outbreak
}

\author{
Marco Di Serafino ${ }^{1}$ (1) $\cdot$ Maria Notaro ${ }^{2} \cdot$ Gaetano Rea $^{3} \cdot$ Francesca lacobellis $^{1} \cdot$ Venere Delli Paoli ${ }^{4} \cdot$ Ciro Acampora $^{1}$. \\ Stefania lanniello ${ }^{5}$. Luca Brunese ${ }^{6} \cdot$ Luigia Romano $^{1} \cdot$ Gianfranco Vallone $^{6}$
}

Received: 8 April 2020 / Accepted: 25 May 2020 / Published online: 13 June 2020

(c) Italian Society of Medical Radiology 2020

\begin{abstract}
Ultrasound is the most disruptive innovation in intensive care life, above all in this time, with a high diagnostic value when applied appropriately. In recent years, point-of-care lung ultrasound has gained significant popularity as a diagnostic tool in the acutely dyspnoeic patients. In the era of Sars-CoV-2 outbreak, lung ultrasound seems to be strongly adapting to the follow-up for lung involvement of patients with ascertaining infections, till to be used, in our opinion emblematically, as a screening test in suspected patients at the emergency triage or at home medical visit. In this brief review, we discuss the lung ultrasound dichotomy, certainties and uncertainties, describing its potential role in validated clinical contexts, as a clinicaldependent exam, its limits and pitfalls in a generic and off-label clinical context, as a virtual anatomical-dependent exam, and its effects on the clinical management of patients with COVID-19.
\end{abstract}

Keywords Lung ultrasound · Point of care ultrasound · Dyspnoea · COVID-19 · Sars-CoV-2

Abbreviatio
ARDS
BLUE
CAUSE
CHP
COPD
CT

Marco Di Serafino, Maria Notaro, Gaetano Rea and Francesca Iacobellis contributed equally to this work.

Marco Di Serafino

marco.diserafino@aocardarelli.it

1 Department of General and Emergency Radiology, “Antonio Cardarelli" Hospital, Naples, Italy

2 Department of Intensive Care Major Burns and Poison Control Center, "Antonio Cardarelli” Hospital, Naples, Italy

3 Department of Radiology, "Vincenzo Monaldi", dei Colli Hospital, Naples, Italy

4 Department of Emergency, "Antonio Cardarelli” Hospital, Naples, Italy

5 Department of Emergency Radiology, "San Camillo" Hospital, Rome, Italy

6 Department of Life and Health, "Vincenzo Tiberio", University of Molise, Campobasso, Italy

\author{
CXR Chest X-ray \\ COVID-19 Coronavirus disease 19 \\ ECMO Extra-corporeal membrane oxygenation \\ EVD Ebola virus disease \\ FALLS Fluid administration limited by lung \\ sonography \\ LUS Lung ultrasound \\ PEEP Positive end-expiratory pressure \\ POC Point of care \\ PPE Personal protective equipment \\ RDA Ring down artifacts \\ SARS-COV-2 Severe acute respiratory syndrome coro- \\ navirus 2
}

\section{Introduction}

Ultrasound is the most disruptive innovation in intensive care life, above all in this time, with a high diagnostic value when applied appropriately, virtually free from the damaging biological effects caused by applying ionizing radiation. Furthermore, the portability of the equipment, which allows for even bedside examination, the comfort of the patient, repeatability, and reduced cost are the true strength of this imaging technique. 
In recent years, point-of-care (POC) lung ultrasound (LUS) has gained significant popularity as a diagnostic tool in the acutely dyspnoeic patients. Patricia Henwood, in her heartfelt experience during the epidemic Ebola outbreak in West Africa, perfectly summarizes the potential role of LUS in critically ill patients: "half my patient died. They died from one disease, but so many different deaths. After a decade of honing my ability to quickly determine "sick or not sick" and allocating time and resources accordingly, I learned that when managing a ward of patients with Ebola, clinical appearance did not always predict survival. Layers of impermeable and stifling personal protective equipment (PPE) constitute an enormous physical barrier to patient care, complicating management of Ebola virus disease (EVD). The ability to auscultate is gone, you cannot smell, and layers of gloves blunt your tactile sense. You cannot risk touching your face to readjust your PPE, so visual cues can also be a challenge $[\ldots]$. We lacked on-site diagnostic capacity, so as I worked to secure critical resources to improve over all care, I also sought and received approval to incorporate point-of-care ultrasonography" [1]. These claims are so much actually in these days of Severe Acute Respiratory Syndrome Coronavirus 2 (SARS-COV-2) pandemic spread so that LUS seems to be strongly adapting to the follow-up for lung involvement of patients with ascertaining Coronavirus Disease 19 (COVID-19) up to, in our opinion emblematic, use of LUS at the emergency triage or at home medical visit as a screening imaging test of suspected COVID-19 patients [2-4]. If on one hand, many studies have shown the efficacy of LUS in diagnosing pulmonary pathology, with increased sensitivity compared to that of chest X-ray (CXR) [5-7], on the other hand, little is discussed about its limits and pitfalls especially in generic and off label clinical contest. Thus, in the brief review, we discuss the LUS of the facts, that is often substance in the critical clinical context (clinical-dependent exam), and the LUS of the artifacts that, equally often, may become only imagination in a generic or inappropriate clinical context (virtual anatomical dependent exam).

\section{Lung ultrasound clinical-dependent exam: many facts}

Several diseases can be detected at the bedside with POC LUS with several clinical implications and management, especially in critically ill patients [8]. The sensitivity of LUS for diagnosis of pulmonary oedema, in some literature articles, approaches $94 \%$, with a specificity of $92 \%$ [9]. Diagnoses of chronic obstructive pulmonary disease (COPD), asthma, pulmonary embolism, pneumothorax, and pneumonia can also be made with POC LUS with a positive predictive value ranging from 83 to $100 \%$ (Table 1) [10,

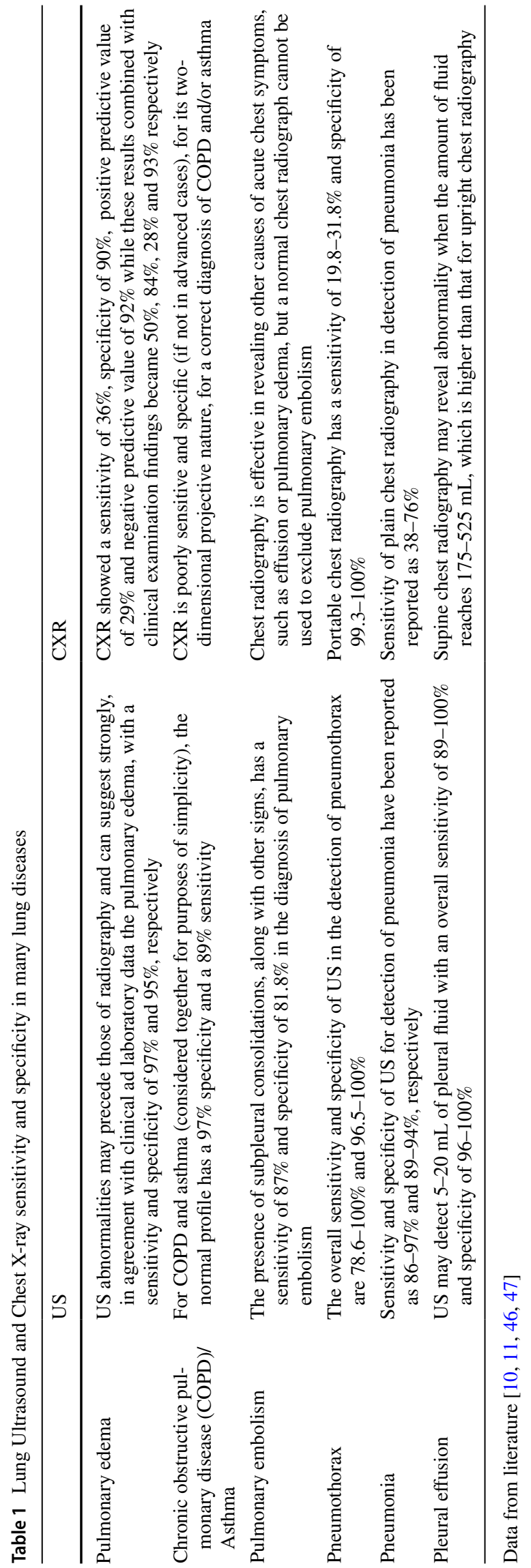


11]. By evaluating 8 zones of the chest -4 on each side (2 anterior and 2 lateral) (Fig. 1a), using both low frequency and high-frequency probes with the best combination of sector or curved array probe $(3.5-5 \mathrm{MHz})$ and a small-parts linear probe (5-10 MHz), an assessment for various pathologies can be made by examining the pleural line (sliding, thickness, regularity), the sub-pleural regions (presence or absence of echogenic changes) and whether there are A-lines or B-lines present (with the amount and location of B-lines assessed) (Table 2) [12].
The majority of acute respiratory disorders involve the pleura, and therefore are accessible to LUS that is a surface imaging technique $[8,13]$. The LUS artifacts come from the pleura, so the pleural line must be detected to avoid mistakes [13]. The hyperechoic pleural line (Fig. 1b, c), constituted by the summation of parietal pleura, pleural space, and visceral pleura, it refers to an artifact generated between tissues with different acoustic impedance, as soft subcutaneous tissues and air underneath the lung [14]. This hyperechoic line is usually motile and it is synchronized with respiratory activity. Such movements are linked to pleural sliding. The

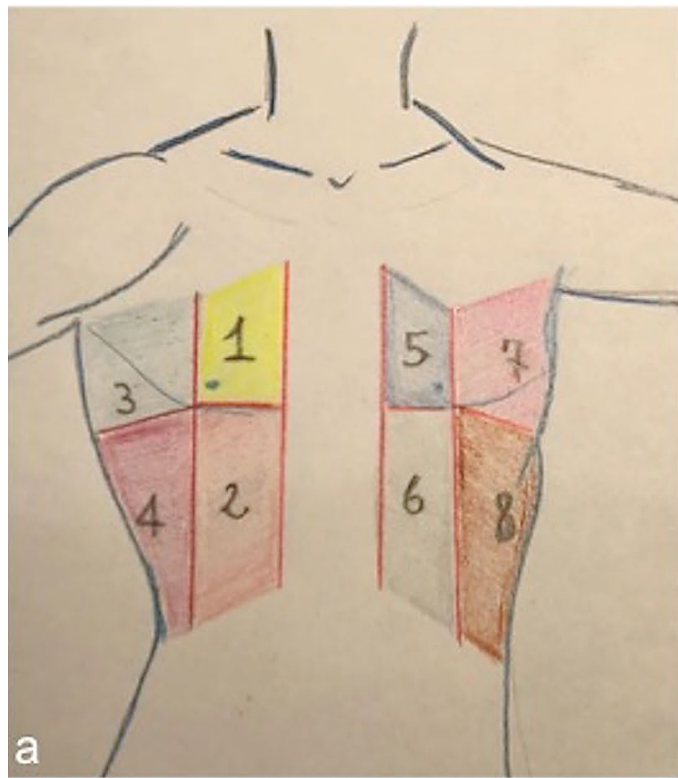

Fig. 1 Schematic representation of the chest ultrasound zone (a) and chest ultrasound examination performed with a high-frequency linear probe $(15-7 \mathrm{MHz})(\mathbf{b}, \mathbf{c}) .8$ zones of the chest -4 on each side (2 anterior and 2 lateral) (a): the anterior zones $(1,2,5,6)$ are delimited medially by the hemi-clavicular line and laterally by the anterior axillary line whereas the lateral ones $(3,4,7,8)$ are included between the anterior and posterior axillary lines. The sub-mammary line divides

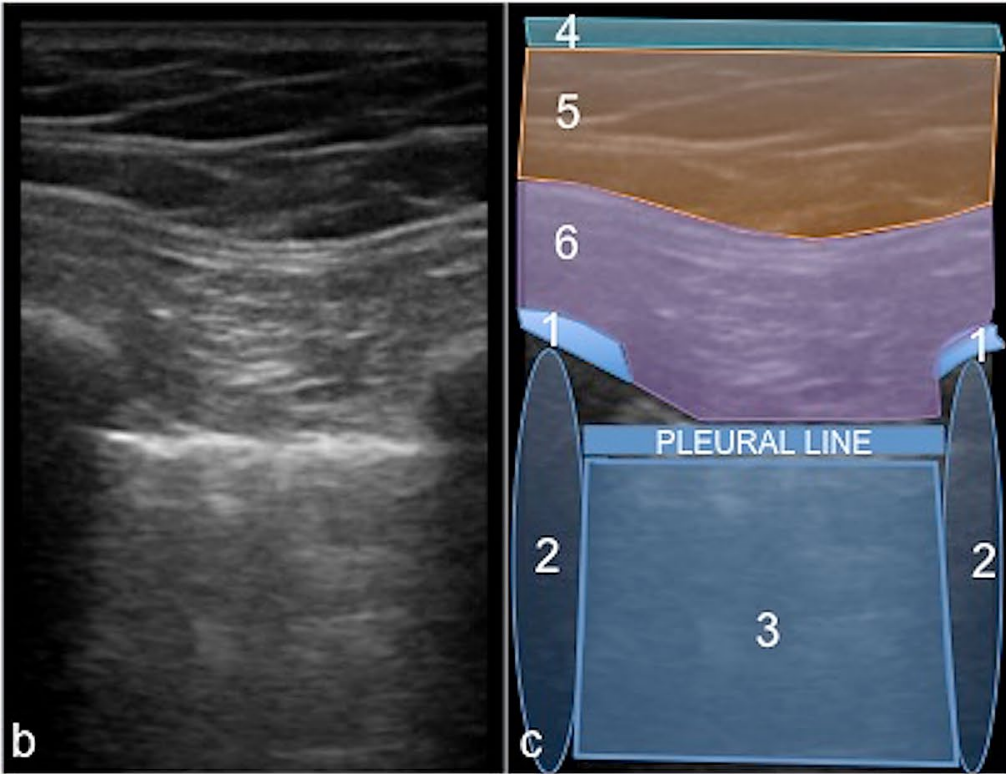

the upper and lower zones. Thoracic anatomy, longitudinal view acquired with linear probe (b), and schematic representation (c): there is a good anatomical definition of the pleural hyperechoic reflection (pleural line, c) between the two ribs (c, 1) and their shadow cone artifacts (c, 2). They outline an area of sub-pleural pulmonary artifacts (c, 3). The cutaneous (c, 4), subcutaneous (c, 5) and muscular planes $(\mathbf{c}, 6)$ are well represented

Table 2 The alphabet of thoracic ultrasound lines

\begin{tabular}{ll}
\hline Type & Findings \\
\hline $\begin{array}{l}\text { Lung and pleural lines } \\
\text { A-line (reverberation) } \\
\text { B-line (ring down artifact) }\end{array}$ & $\begin{array}{l}\text { Hertical hyperechoic artifact originating from the pleural line that extend to the edge of the screen, follow the lung } \\
\text { sliding and erase the A-lines } \\
\text { Short, ill-defined vertical hyperechoic lines arising from the pleural line that do not reach the edge of the screen } \\
\text { nor erase the A-lines nor follow the lung sliding } \\
\text { Short, triangular artifact with caudal apex lines arising from the pleural line following the lung sliding without } \\
\text { reaching the edge of the screen }\end{array}$ \\
Superficial lines & Vertical hyperechoic artifact arising superficial to and obscuring the pleural line and extending to the edge of the \\
E-line & screen
\end{tabular}


Table 3 Artifacts dichotomy

\begin{tabular}{|c|c|c|c|c|c|c|c|}
\hline \multicolumn{2}{|l|}{ A-lines } & \multicolumn{2}{|l|}{ B-lines } & \multicolumn{2}{|c|}{ Z-lines and comet tail } & \multicolumn{2}{|l|}{ Lung sliding } \\
\hline Normal & Abnormal & Normal & Abnormal & Normal & Abnormal & Normal & Abnormal \\
\hline Healthy patients & $\begin{array}{l}\text { Atelectasis } \\
\text { Asthma } \\
\text { COPD } \\
\text { Pneumothorax }\end{array}$ & Normal finding & $\begin{array}{l}\text { Degrees of interstitial } \\
\text { disease } \\
\text { Pneumonia } \\
\text { Pneumothorax (possible) }\end{array}$ & Normal finding & Also present & $\begin{array}{l}\text { Healthy patients } \\
\text { Lung pulse }\end{array}$ & $\begin{array}{l}\text { Pneumothorax } \\
\text { Apnoea }\end{array}$ \\
\hline
\end{tabular}

"gliding sign" or "lung sliding" can indicate the absence of pneumothorax with accuracy close to $100 \%$ (Table 3) [15-17]. The "lung pulse", described initially in 2003 by Lichtenstein et al. is a dynamic LUS sign refers to the rhythmic movement of the pleura in synchrony with the cardiac rhythm. It is best viewed in areas of the lung adjacent to the heart, at the pleural line due to cardiac vibrations transmitted to the lung pleura in poorly aerated lung and it is an early ultrasound sign of complete atelectasis or consolidation as well as indirectly exclude pneumothorax in absence of lung sliding in this critical point (Table 3). In normal well-aerated lung, the 'lung pulse' is not present, as lung sliding becomes dominant and resistant to cardiac vibrations $[17,18]$.

LUS semeiotic include also other artifacts derived by air/ tissue interface such as horizontal and longitudinal/vertical reverberations. The horizontal reverberations, known as A-lines, are multiple repeating horizontal lines that are parallel and equidistant from the pleura (Fig. 2a, b) (Table 2); these are the result of repeated reflections of the linear hyperechoic structure caudally to the pleural hyperechoic line, usually interpreted as a normal finding in the healthy patient (Table 3). However, A-lines can also be prominent
Fig. 2 Lung ultrasound examination performed with a high-frequency linear probe (15-7 MHz) in healthy adults (a-d), longitudinal view.

A-lines (a) with schematic representation (b): there is a good anatomical definition of the pleural hyperechoic reflection (b thickened blue line) with evidence of multiple horizontal reverberation artifacts or A-lines (b, transparent blue boxes) parallel to the hyperechoic "pleural line" and equidistant between them. B-lines (c) with schematic representation $(\mathbf{d})$ : multiple hyperechogenic linear structures or ring-down artifacts (c) come from the hyperechoic pleural line (d thickened blue line) caudally for several $\mathrm{cm}$ (d blue vertical trapezoids)
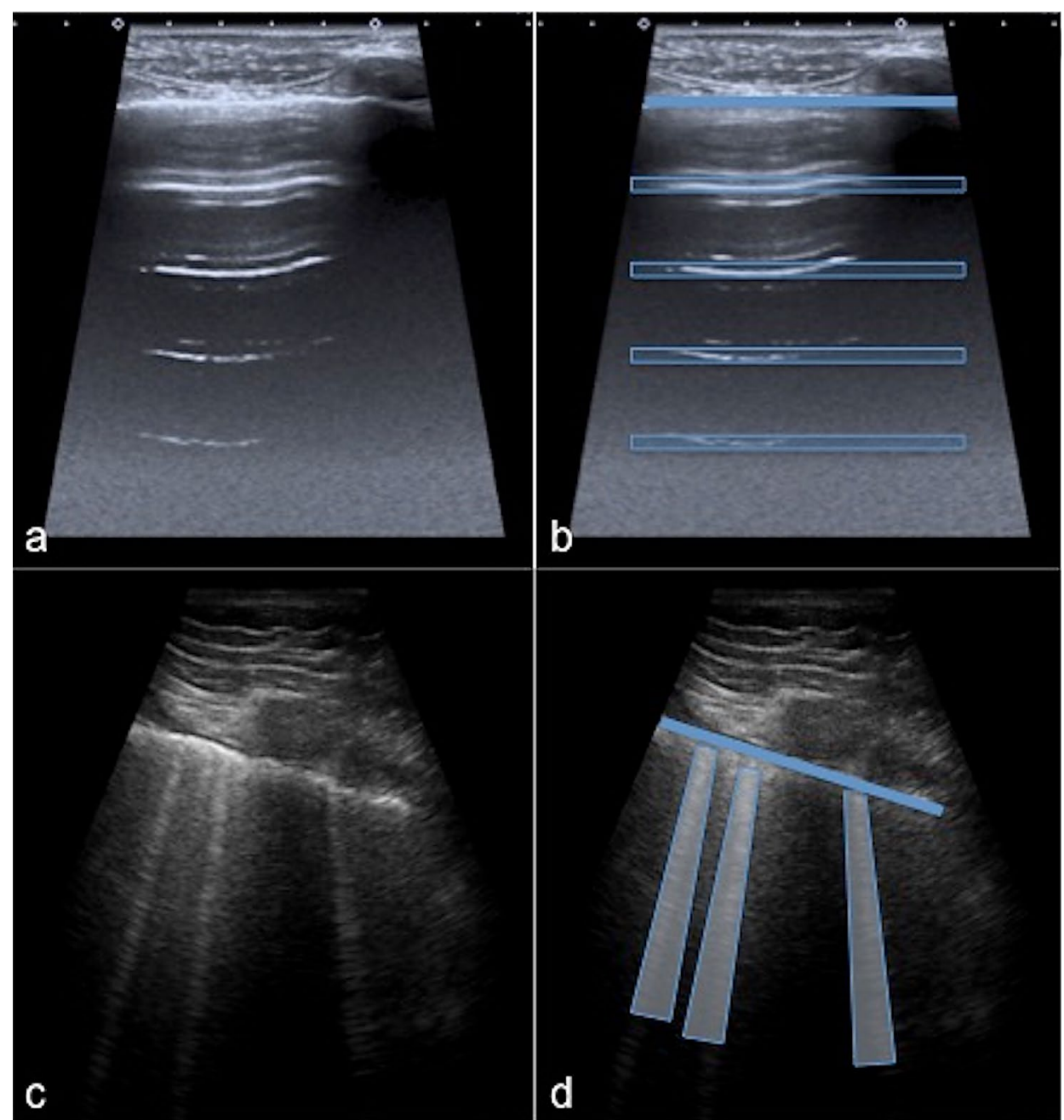
in patients with atelectasis, asthma, COPD (positive lung sliding at the pleural line), and pneumothorax (negative lung sliding at the pleural line) (Table 2) [14-16]. Longitudinal/ vertical reverberations, known as B-lines or ring-down artifacts (RDA), are transient, hyperechoic vertical lines that extend from the pleura to the bottom of the screen, at a maximum depth of $16 \mathrm{~cm}$, erasing A-lines and moving in concert with lung sliding (Fig. 2c, d) (Table 2). Although also present in healthy subjects, three or more B lines in any given region usually represent a pathologic finding (Table 3 ). In the case, three or more B-lines between two ribs are called "lung-rockets" and correlate with the interstitial syndrome with $93 \%$ accuracy using alveolar-interstitial radiographic changes as a reference, and full accuracy using computed tomography (CT) [8]. Up to 3 B-lines are called "septal rockets", correlated with Kerley B-lines at chest-X-ray that represents thickened interlobular septa during the interstitial syndrome (Fig. 3a, b) [8]. Twice as many, called "groundglass rockets", correlate with ground-glass areas (Fig. 3c, d) [8]. In general, in validated clinical contexts, focal B-lines arranged peripherally to a consolidation area may suggest pneumonia, whereas diffuse B-lines in three or more zones on both sides of the chest suggests a diffuse alveolar interstitial syndrome such as pulmonary oedema or acute respiratory distress syndrome (ARDS) (Fig. 4) (Table 3) [8-16]. Furthermore LUS semeiotic is also composed of "anatomical" real images that can also coexist in different clinical contexts such as pleural effusions and sub-pleural consolidations (tissue-like sign) with "static" or "dynamic" air bronchogram (Fig. 5). The "dynamic" air bronchogram could indicate pneumonia in some clinical setting, distinguishing it from resorptive atelectasis. Otherwise, static air bronchograms correlate better with most resorptive atelectasis and only one-third of cases with pneumonia [19].

The evaluation of diaphragmatic excursions can be an useful tool in ventilated patients as well as its indisputable additional role as a guide during interventional biopsy or drainage procedures too [8-16]. All these findings are well summarized in the management of critically ill patients through an accurate clinical synthesis that is a pivotal element in some validated LUS protocols such as [20]:
Fig. 3 Chest ultrasound examination performed with a high-frequency linear probe $(15-7 \mathrm{MHz})$ in an adult patient with heart failure (a-d). Up to 3 B-lines (a) with schematic representation (b) in an interstitial congestive heart failure: multiple hyperechoic linear structures or ring-down artifacts (a) in a septal rockets configuration come from the hyperechoic pleural line (b thickened blue line) caudally for several $\mathrm{cm}$ (b blue vertical trapezoids). Many B-lines (c) with schematic representation (d) in an alveolar-interstitial congestive heart failure (same patient as before at follow-up): multiple hyperechogenic linear structures or ring-down artifacts (c) in a ground-glass rockets configuration come from the hyperechoic pleural line (d thickened blue line) caudally for several $\mathrm{cm}$ (d blue vertical trapezoids)
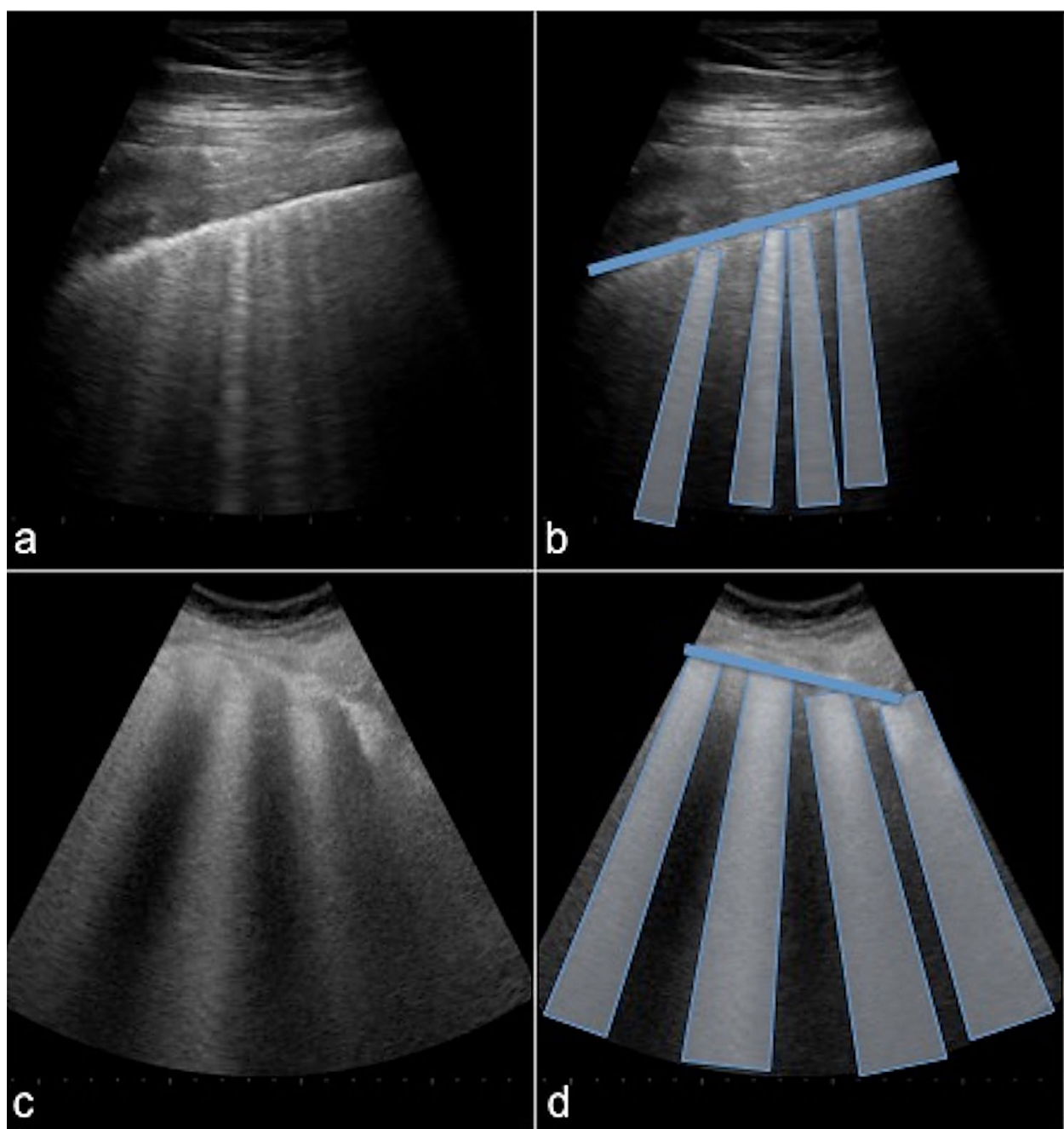


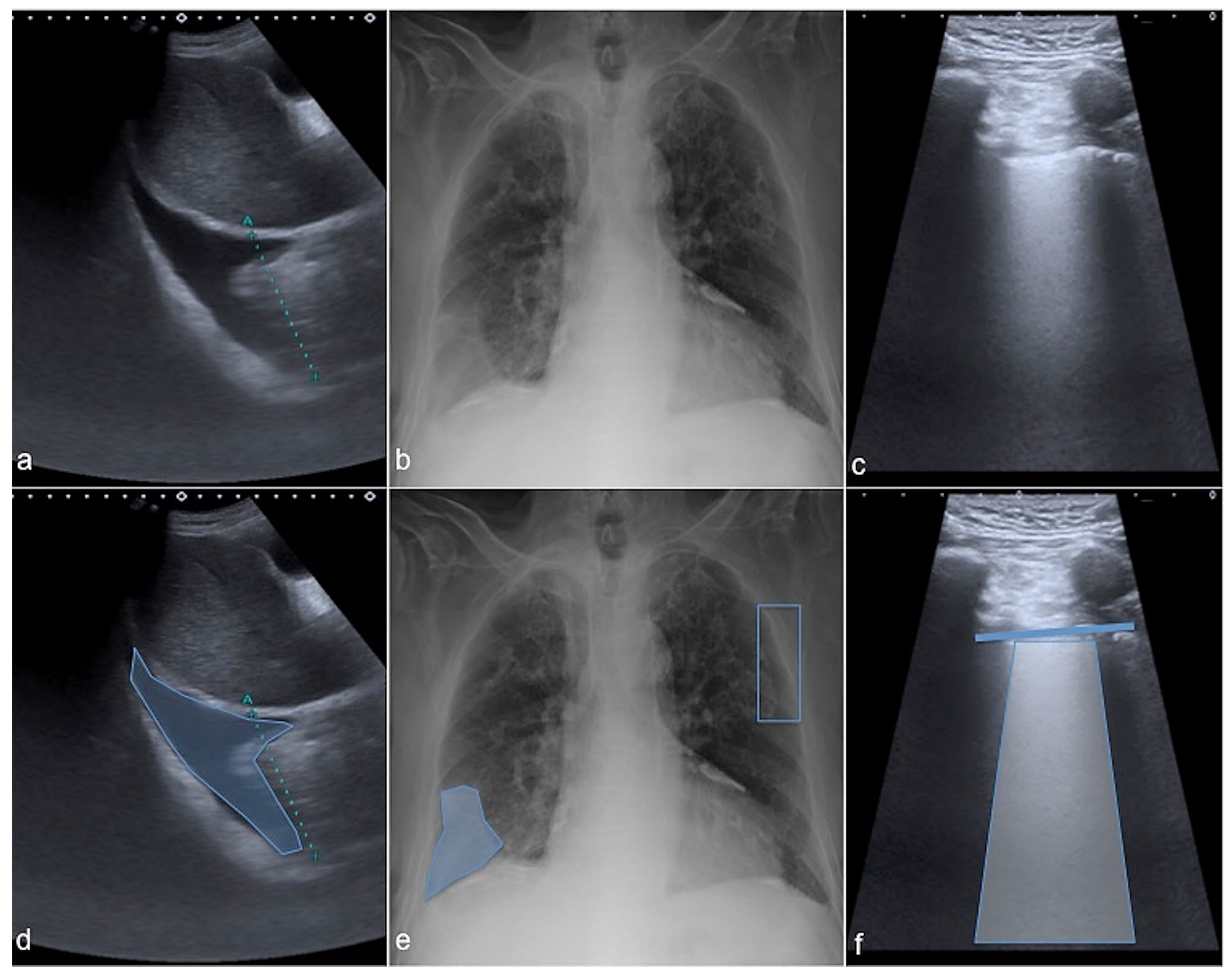

Fig. 4 Integrated ultrasound (a, c) and chest X-ray (b) with schematic representation (d, e, f) of an elderly patient admitted for acute decompensated heart failure: the opacity at the right base on chest X-ray (b, e-blue area) corresponds to a pleural effusion on lung ultrasound (a, $\mathbf{d}$-blue area); a ground-glass rockets configuration (c, $\mathbf{f}-$ blue verti- cal trapezoid; pleural line: thickened blue line) was also observed in all lung fields (b, e). The blue box (e) represents a detail of the lung ultrasound scan $(\mathbf{c}, \mathbf{f})$, with the probe longitudinally placed. The integrated imaging orients for interstitial-alveolar oedema
Fig. 5 Chest ultrasound examination performed with a low-frequency convex probe (5-10 MHz). Consolidative area (a) with schematic representation (b) in bacterial pneumonia: the tissue like sign (b blue area) with hyperechoic spots inside (b yellow arrowheads)

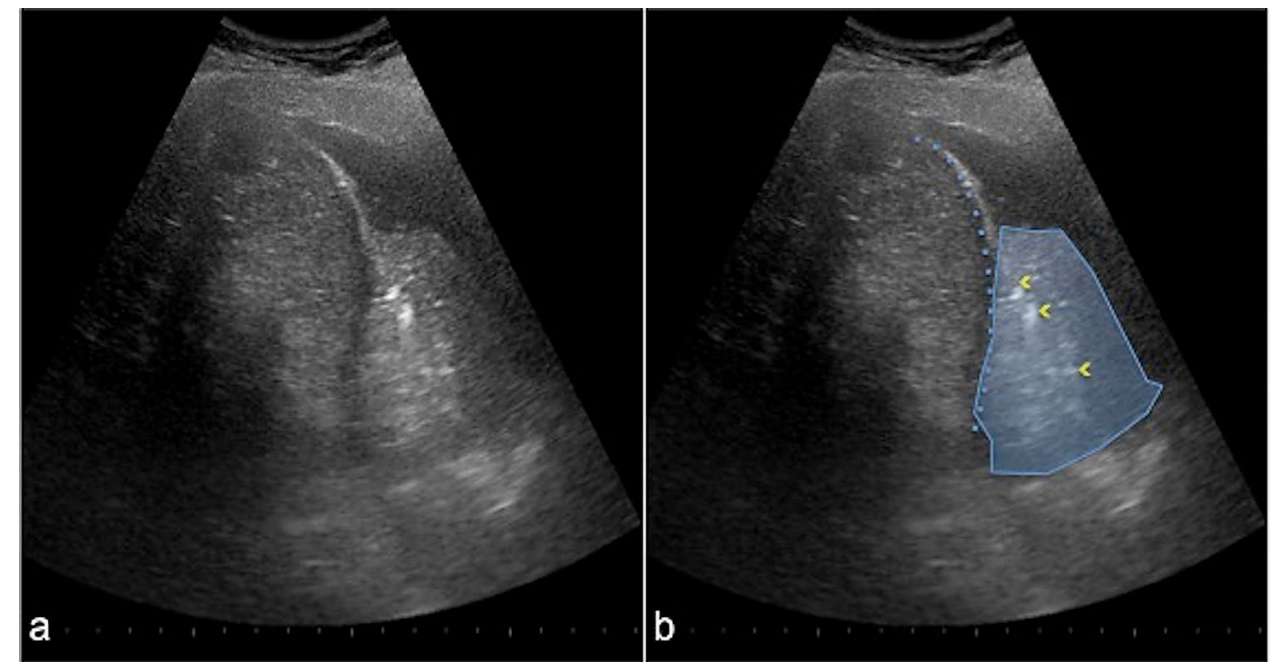


- BLUE protocol (Bedside Lung Ultrasonography in Emergency) $[8,21]$ : emergency protocol for immediate diagnosis of acute respiratory failure. This protocol describes specific sonographic findings associated with major conditions, such as pneumonia, congestive heart failure, chronic obstructive pulmonary disease, asthma, pulmonary embolism, and pneumothorax, with more than $90 \%$ diagnostic accuracy. It consists in the identification of ten signs: the pleural line; the lung sliding; the A-lines (horizontal artifact); the pleural effusion; the tissue-like sign indicating lung consolidation; the B-lines and lung rockets indicating interstitial syndromes; abolished lung sliding suggesting pneumothorax and the lung point with the absence of B-lines and lung pulse indicating with pneumothorax. Two more signs, the lung pulse, and the dynamic air bronchogram, are used to distinguish atelectasis from pneumonia.

- FALLS protocol (Fluid Administration Limited by Lung Sonography) [8, 21]: emergency protocol designed to sequentially rule out differential diagnoses such as cardiogenic and hypovolemic shock, and allowing an early diagnosis of septic shock. It adapts the BLUE-protocol to acute circulatory failure and consists in studying the change from A-lines to lung rockets at a threshold of $18 \mathrm{~mm} \mathrm{Hg}$ of pulmonary artery occlusion pressure, providing a direct biomarker of clinical blood volume. The appearance of B-lines, schematically, is considered as the endpoint for fluid therapy.

- CAUSE protocol (Cardiac Arrest Ultrasound Exam) protocol [13, 22]: this protocol normalizes the use of ultrasonography in cardiac arrest management. It has the potential to reduce the time required to determine the etiology of a cardiac arrest and thus decrease the time between arrest and appropriate therapy. This approach, incorporating LUS to manage a cardiac arrest, aids in the diagnosis of the most common and easily reversible non-cardiac causes of arrest, such as severe hypovolemia, tension pneumothorax, cardiac tamponade, and massive pulmonary embolus.

Furthermore, LUS can be also used as a diagnostic tool for assessing positive end-expiratory pressure (PEEP), induced lung recruitment. It can allow close monitoring of any respiratory manoeuvers aimed to improve lung aeration [13, 23]. LUS may also be used to assess and monitor lung aeration in a patient with ARDS. An increase in lung aeration resulting from prone positioning was detected using LUS [24]. LUS permits also an ultrasound dynamic assessment of lung injury in a patient with extra-corporeal membrane oxygenation (ECMO) [25].

\section{Lung ultrasound "virtual anatomic" dependent exam: only artifacts}

As seen, although the axiom that LUS is something impossible is now abundantly overcome, it is equally important strongly affirm that LUS has such intrinsic, patients and radiologists/other specialists correlate limits, and therefore its ultra-diagnostic use with an "everything and anything" imperative can similarly be a dangerous claim: the truth of LUS is in the middle. Some fine diagnoses still need a more detailed approach as well as a high-resolution CT scan [26].

\section{LUS intrinsic limitations}

LUS, in optimal conditions, assesses only the $70 \%$ of lung surface, which is only about $1 / 16$ of the total lung, due to the anatomical constraints of the thoracic cage, and even in areas subject to a LUS examination, only the alterations closely related to the pleural surface may be visualized (Fig. 6) [27]. This condition partly explains the rather low sensitivity of LUS to detect intra-parenchymal pneumonia, not-adherent to the pleural surface such as some consolidations or tumours, that can be medially located and surrounded by the aerated
Fig. 6 Lung field of view on CT (a) compared with ultrasound (b). The blue box (a) schematizes the ultrasound field of view (b) in relation to the $\mathrm{CT}$

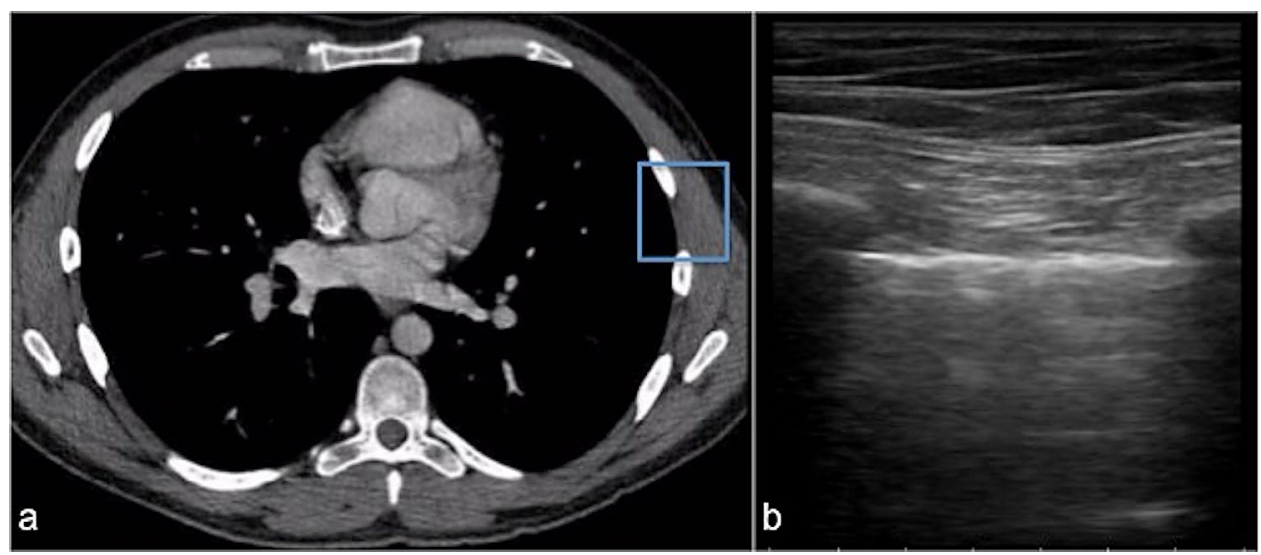


lung, or some pulmonary interstitial syndrome from different aetiologies, which may spare the subpleural space $[27,28]$. For this reason, a satisfactory pulmonary evaluation or even higher than the chest radiography cannot be reached by LUS. Indeed, only alterations linked to the pleura can be "seen" and evaluated with the ultrasound probe. Besides, the sensitivity of thoracic ultrasound, in the presence of an alterations that affects the pleural compartment, can be considered high, but the specificity is always discreetly low, as LUS cannot characterize alterations: a pneumonia, a tumour or atelectasis may show the same echographic pattern (Fig. 7) [27]. According to our opinion, only a CT, and sometimes not even a CT, can be considered a certainty investigation to diagnosing a pulmonary alteration, in such cases only a biopsy can confirm the clinical-diagnostic data.

\section{Patients' dependent limitations}

Obese patients may be more difficult to examine due to the thickness of their ribcage and soft tissues, as well as it is difficult to perform LUS in burn patients. The presence of subcutaneous emphysema or large thoracic dressings alters or precludes the propagation of the ultrasound beams to the sub-pleural lung parenchyma [28]. Moreover, the coexistence of several pathologies that increase or reduce the sub-pleural air content such as emphysema or atelectasis respectively or an existing fibrotic interstitial lung disease can be confounding factors in the interpretation of the LUS findings in the acute setting that are also strongly depending on the age of the observed patient [29].

\section{Variability interpretation of the data and confusing findings}

LUS is often considered the imaging methods with a shorttraining course $[8,30]$, and this is largely due to its poor, more intuitive, and not anatomical findings, wrongly understood as a very simple procedure. No clear evidence-based guidelines or recommendations exist on the training needed to obtain adequate skills for performing a LUS examination [30]. The value of LUS is dependent on competent operators performing the examination and the risk of the
Fig. 7 Chest ultrasound convex multi-frequency probe (a) with schematic representation (b) of inferior right lobar pneumonia: inhomogeneous consolidation adhered to the pleura (blue area, b) with infected pleural effusion (circled area, b). Enhanced chest CT (c, mediastinal window) clearly demonstrates drainage tube with a better visualization of the pleural effusion and extensive right lobar consolidation with air and fluid bronchogram in context

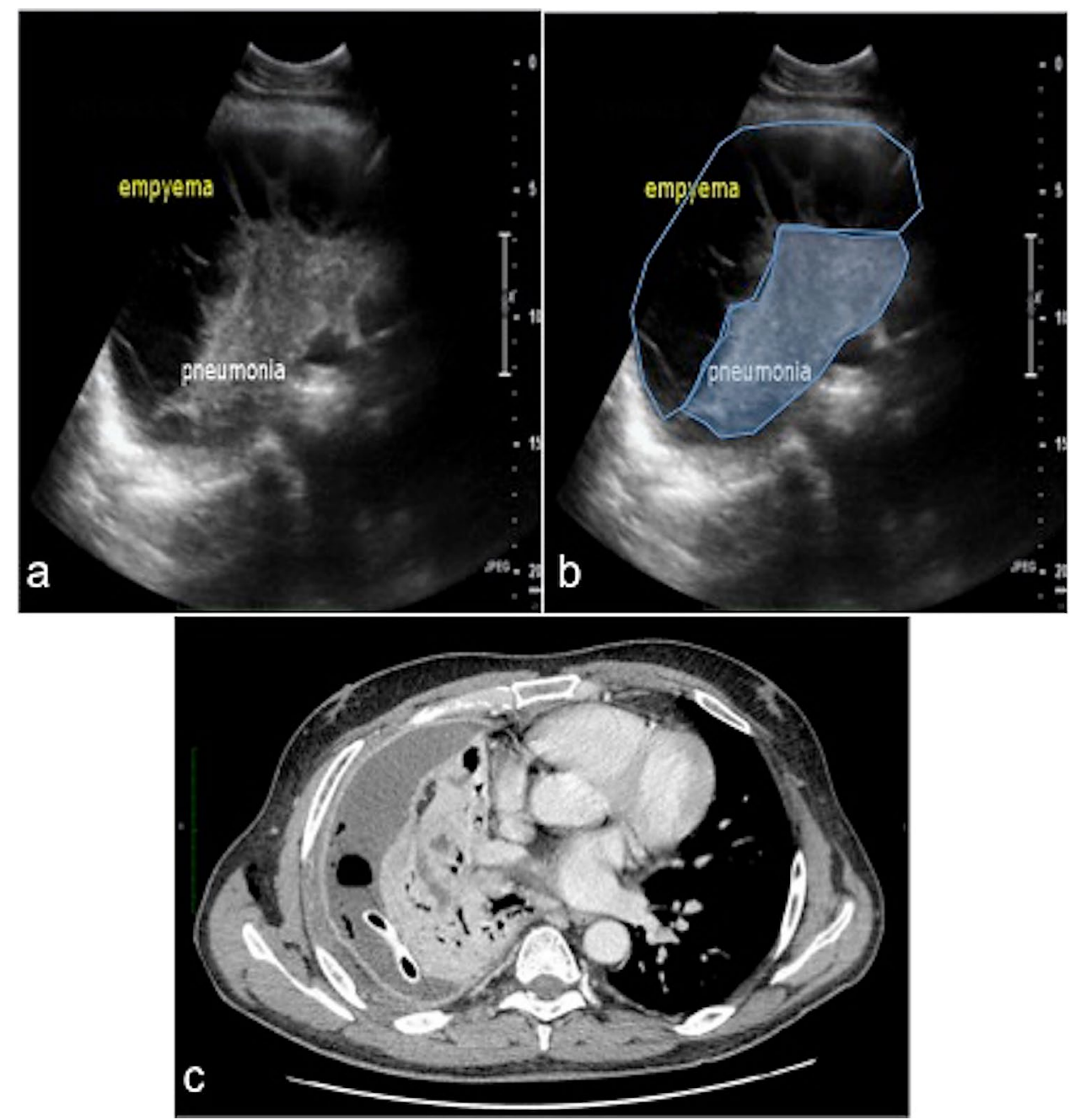


inadequacy of the LUS in inexperienced hands can cause more harm than good. When LUS is used inappropriately by novice or inexperienced physicians, it's findings become easily confounding; for example, the B line are often misdiagnosed as Z-lines which are vertical, band-like echogenic reinforcements, fixed on the lung fields, which do not delete the A-lines without any pathological correlation (Fig. 8) (Tables 2, 3). Furthermore, the interpretation of the B-lines can be subject to severe inter-observer and intra-observer bias [29]. The interstitial syndrome is often defined as more than three B-lines between two ribs, but we just remember that B-lines are not always pathological findings since healthy people have B-lines, and various disease can often create similar ultrasound findings, which are non-specific if there is no adequate clinical, anamnestic and laboratory evaluation (Figs. 9, 10) (Table 3) [26]. The quantification of B-lines and the assessment of the distance between them can be difficult. This appears to be true because the main distance of two adjacent B-lines at the lung surface should be never less than $7 \mathrm{~mm}$ to be significant and not only when B-lines are numerous or tend to merge [32]. Thus, there is a need of a standardized method of quantification, objective and repeatable. Furthermore, despite its apparent simplicity, a number of pitfalls exist in the use of LUS. B-lines can resolve rapidly in response to treatment, and, therefore, LUS data must be interpreted in the context of previous interventions [33, 34]. B-lines can be seen in several of pulmonary conditions, including pulmonary fibrosis or interstitial lung disease, ARDS, trauma, asthma, and pneumonitis (Table 3) [31, 32, 35-39]. However, the coexistence of diffuse B-lines echo-pattern with the presence of heterogeneous lung echo-texture, irregular thickened pleura and
Fig. 8 Chest ultrasound examination performed with a high-frequency linear probe (15-7 MHz). Z-line (a) and B-line (c) with schematic representation (b-d) in two different healthy patient $(\mathbf{a}, \mathbf{c})$ : hyperechogenic linear structures (a arrow) come from the hyperechoic pleural line (b thickened blue line) caudally for few $\mathrm{cm}$ (b transparent blue vertical trapezoid) without deleting the A-lines (b transparent blue box). Otherwise, the B-lines extend deeper than Z-line (d blue vertical trapezoid) and deletes the A-lines (d transparent blue box)

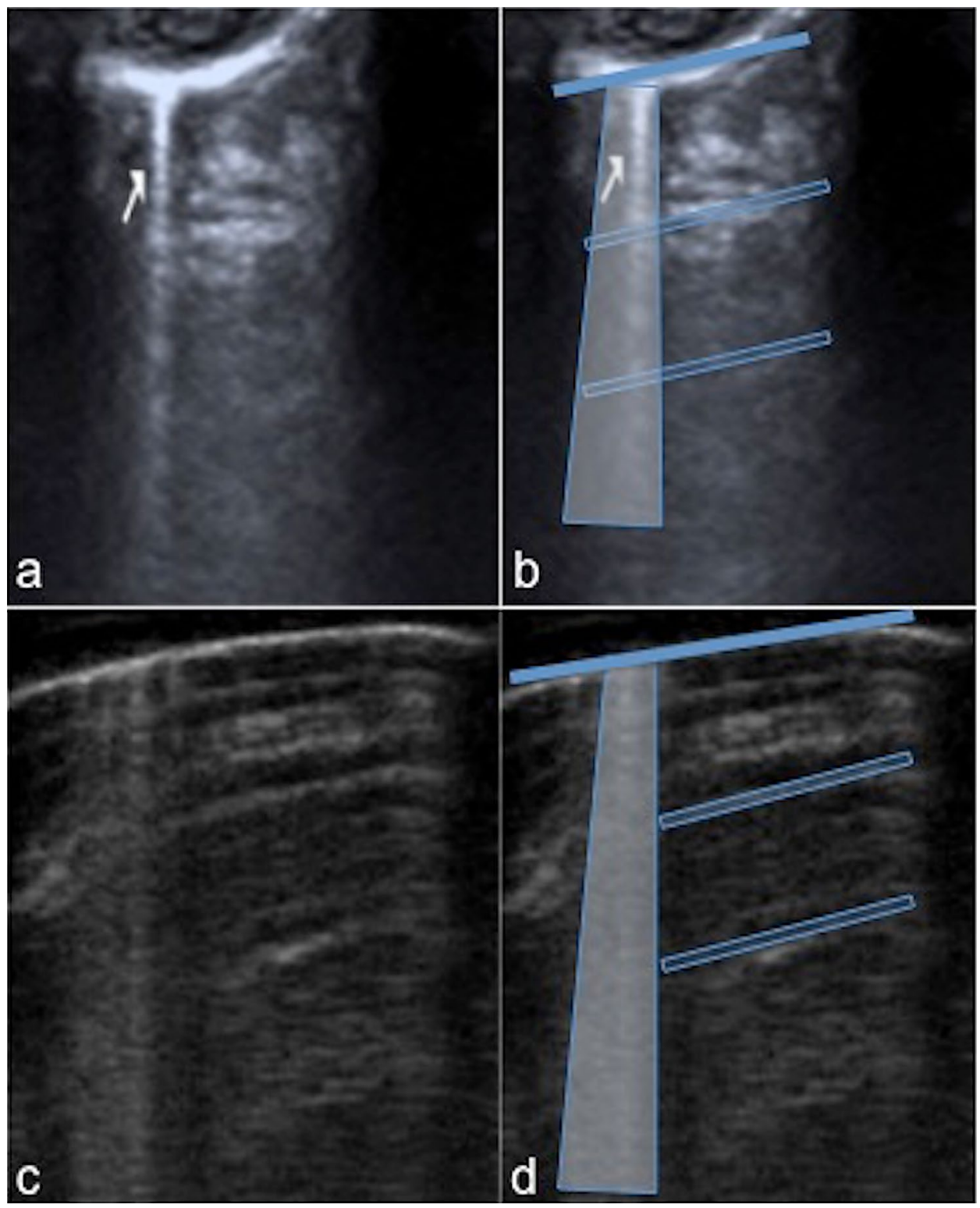




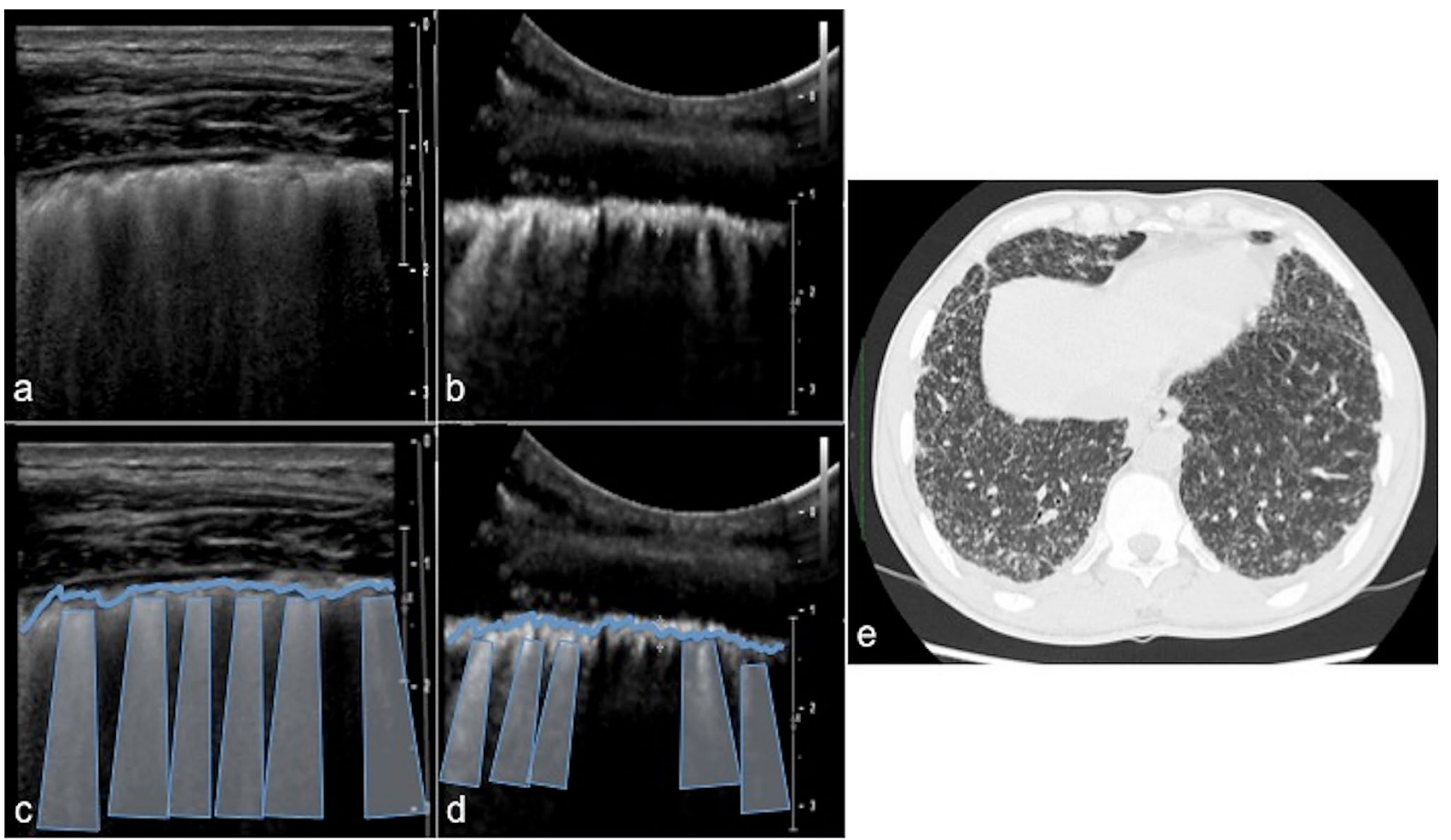

Fig. 9 Chest ultrasound linear multi-frequency probe (a) and convex multi-frequency probe (b) with schematic representation $(\mathbf{c}, \mathbf{d})$ in patient affected by miliary tuberculosis: marked irregularity and notched appearance of pleural line (c-d irregular thickened blue line) with multiple B-lines (c-d blue vertical trapezoids) that realize a non-specific pattern. Chest CT (e lung window) clearly demonstrates multiple very small nodules in both lungs in a patient with miliary tuberculosis presence of scattered "shred sign" could help in diagnosing multifocal pneumonia complicated by ARDS on the related severe degree of hypoxemia, but the ultrasound findings for a consolidation are not specific and must necessarily be correlated with the clinical history, as in the case of aspiration pneumonia (Fig. 11) [40]. The presence of large pleural effusions might interfere with B-line quantification in the affected thoracic zones and induce lung consolidation [32]. Lung consolidations do not necessarily mean pneumonia: hemodynamic pulmonary oedema, pulmonary embolism, all kinds of atelectasis, contusion, tumour, drowning and others generate consolidations [26]. Furthermore there is no clear evidence in the literature that the hyperechoic spots and/ or lines inside consolidations correlate to the CT imaging of air bronchogram nor they can be only considered in the differential diagnosis between atelectasis and inflammation consolidation, since also in the tumours, can be detected the air bronchograms [27]. Misdiagnosis of pneumothorax could be related with abolished lung sliding due to the lung pulse or to patient apnoea. Otherwise, the movement of the air/ pleural effusion interface during the patient's breathing cycle may mimic lung sliding in hydro-pneumothorax (Table 3). Furthermore the B-lines detection does not exclude the presence of the pneumothorax: in fact bubbles placed in the air/effusion interface at the parietal and pleura specially in hydro-pneumothorax may cause a vertical reverberation artifact mimicking a B-line although such artifacts do not originate from the pleural line as the $\mathrm{B}$ lines by definition (Table 3) [41]. Additionally, cases of pneumothorax associated with subcutaneous emphysema cannot be diagnosed by LUS. Indeed, the presence of emphysema in the subcutaneous tissue does not allow the US beam to reach the pleural line, thus preventing the visualization of any image, as well as misunderstanding the so-called E-lines such as the reverberations of air in depth tissue, due to subcutaneous emphysema which extend down to the edge of the screen and erase the pleural line (Table 2). The E-lines should not be confused with B-lines because the latter arise from the pleural line [11, 42].

A correct sequential approach, which could be right for diagnosis of pneumothorax, provides to recognize the A-lines first (anteriorly in supine patients) and then looking for the "lung point" (the junction point between absent pleural sliding with A-lines and presence of pleural sliding with B-lines) (Fig. 12) [26, 41]. For inexperienced physicians, it is common to fall in differential diagnostic pitfalls which are always lurking, such as: pericardial effusion vs. pleural vs. ascites vs. epicardial fat; right ventricle dilation 


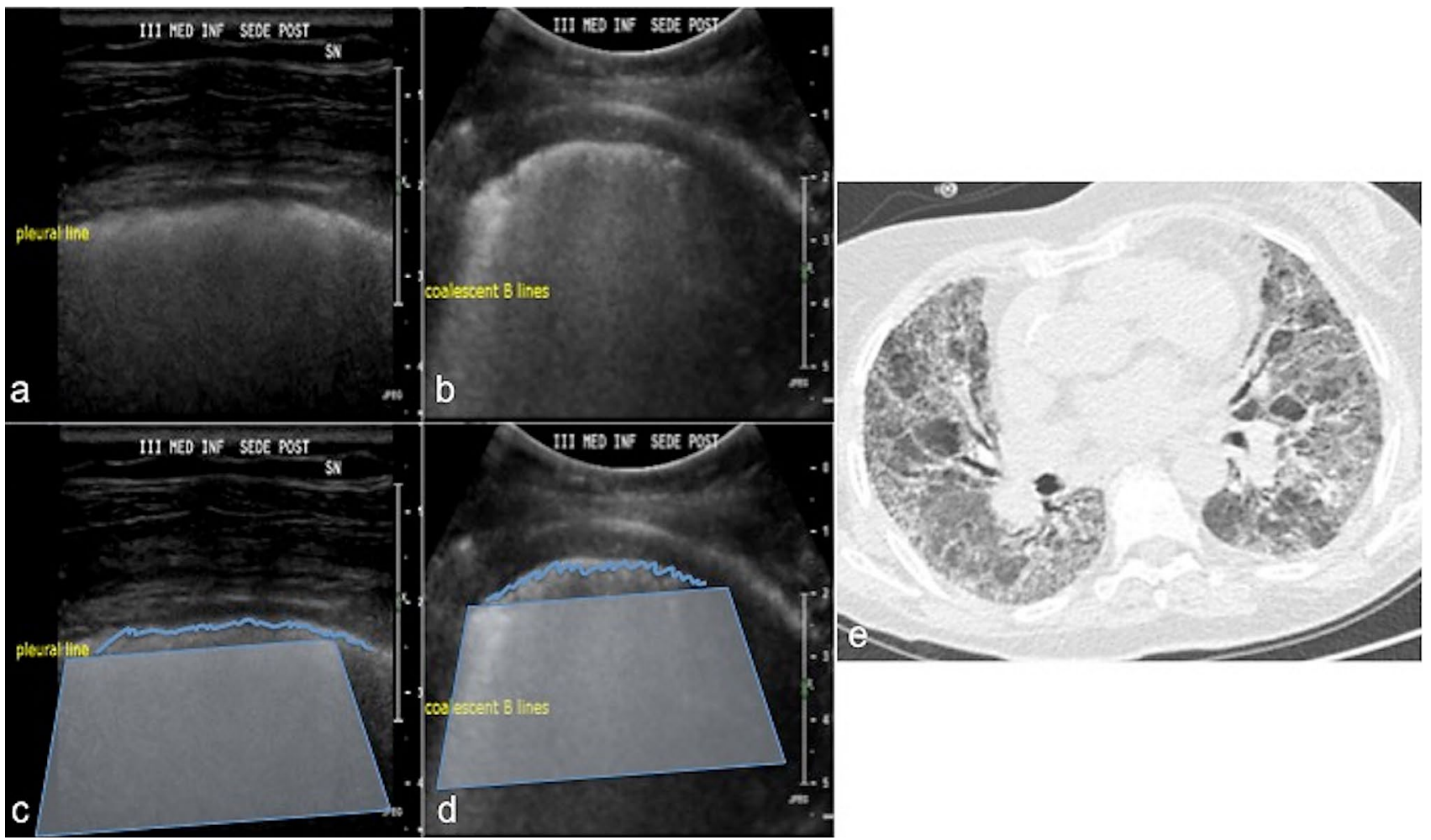

Fig. 10 Chest ultrasound linear multi-frequency probe (a) and convex multi-frequency probe (b) with schematic representation $(\mathbf{c}, \mathbf{d})$ in patient affected by chronic hypersensitivity pneumonia (CHP): marked irregularity and thickened appearance of pleural line (c, d irregular thickened blue line) with multiple B lines (c, $\mathbf{d}$ blue vertical trapezoid) with a "coalescence" appearances that realizes a nonspecific pattern. Chest CT (e lung window) shows the "headcheese

in acute pulmonary embolism and inferior vena cava for volume status assessment in cardiac ultrasound; lung point and lung pulse misinterpretations and mirror artifacts vs. lung consolidations in lung ultrasound (Fig. 13); peritoneal fluid vs. stomach cavity and a critical appraisal of gallbladder signs of acute cholecystitis in abdominal ultrasound; the rouleaux phenomenon vs. deep vein thrombosis or acute right strain in vascular ultrasound [43].

The training and advanced study of ultrasound techniques, thorough knowledge of human anatomy, the study of US artifacts, and ultrasonographic semiotics are all crucial tools for radiologists and other physicians. This is particularly true because the availability of quality and portable ultrasound machines, often used in intensive care units, has led to the application of various techniques in the investigation of a given pathology by professionals from different backgrounds [14]. Furthermore, the lack of specific guidelines has caused diagnoses to be reported in unclear and inconsequent ways, this endangering pattern' in CHP. Ground-glass opacities (high-attenuation areas) are simultaneously highlighted together with less affected parenchyma (low-attenuation areas). It is also visible thickening of the interlobular septa in subpleural space. The imaging pattern reflects variable lung attenuation that results in a heterogeneous appearance of the parenchyma

patients' management. Such risks have been amplified by an increase in borderline and off-label approaches to the study of pleuro-pulmonary pathology [14].

\section{Confounding terminology}

The term "comet tail" artifact in LUS, as such as, has caused confusion simply because it is used to describe any vertical artifact, particularly B-lines, otherwise defined as RDA (Table 2). As already specified by Lichtenstein, in a critical review of LUS for expertise, "the comet-tail artifact does not exist" being itself an oxymoron [26]. The two artifacts present very different physical connotations and ultrasound findings. Comet tail artifact are extremely reduced in size (3-5 mm) [13]. Unlike B-lines, they immediately appear caudally to the hyperechoic pleural line, showing triangular morphology and a caudal apex [14]. Comet tails never reach the caudal distal portion of the echotomographic screen and move in sync with respiratory movements (Figs. 14a, 


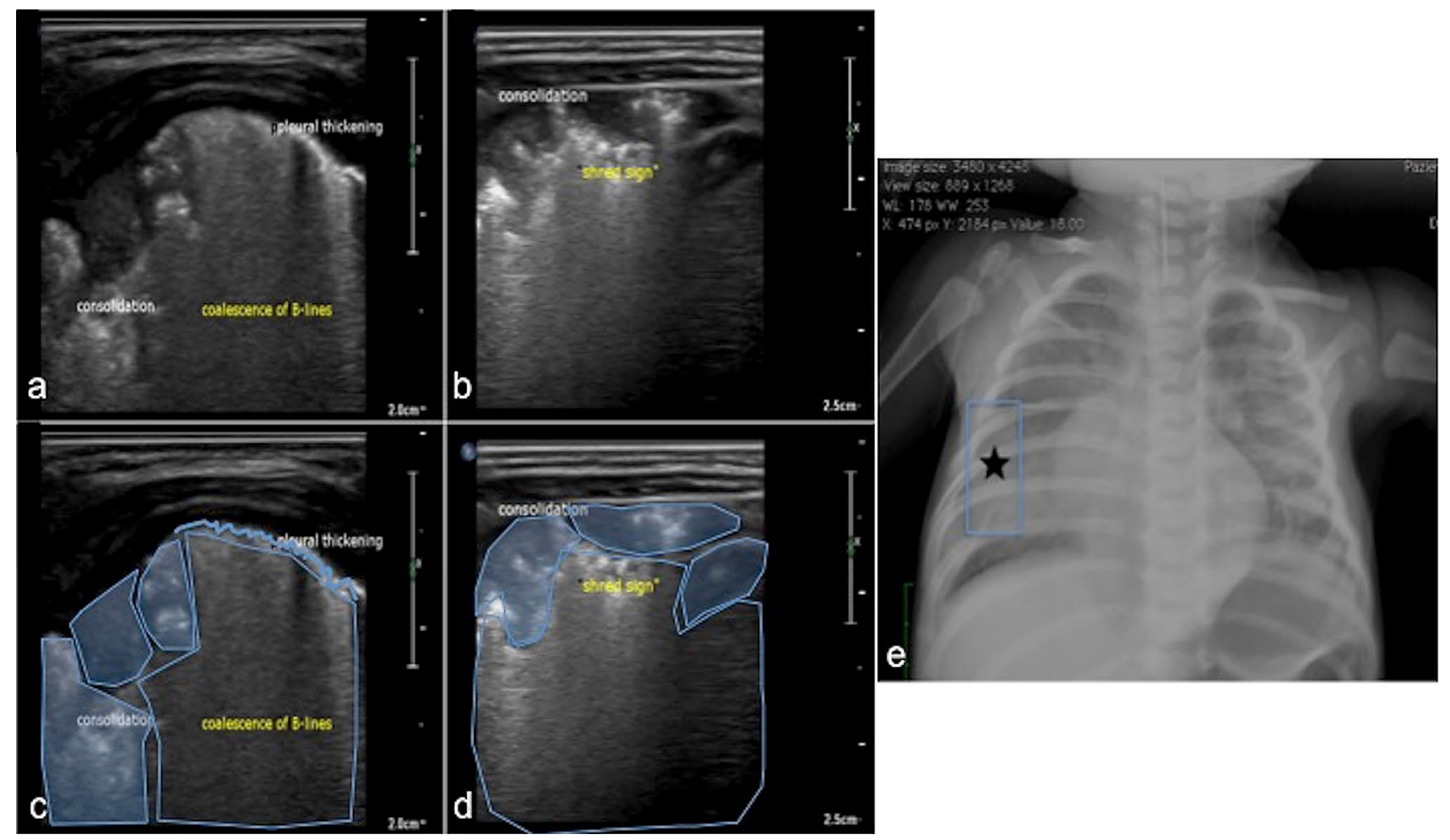

Fig. 11 Chest ultrasound linear multi-frequency probe $(\mathbf{a}, \mathbf{b})$ with schematic representation (c, d) and chest X-ray (e) in patient affected by respiratory distress syndrome: on the left (a) inhomogeneous consolidation (c transparent blue areas) adhered to the pleura with multiple B-lines in "coalescence aspect" (c circled area); on the right (b) multiple small consolidation (d blue areas) closely connected to the pleura in aspiration pneumonia (milk) with shred sign (d circled area). Chest X-ray shows extensive opacity in right lower filed (e blue box) related to aspiration pneumonia (milk) in Down syndrome
Fig. 12 Chest ultrasound examination performed with a high-frequency linear probe (15-7 MHz). Pneumothorax (a) with schematic representation (b): normal pleural lung sliding (double heads thickened arrow) with B-lines (b blue vertical trapezoids) and a lung point (b bolt) with fixed pleura (b thickened line) due to pneumothorax; the A-lines (b blue box) underlies the pneumothorax

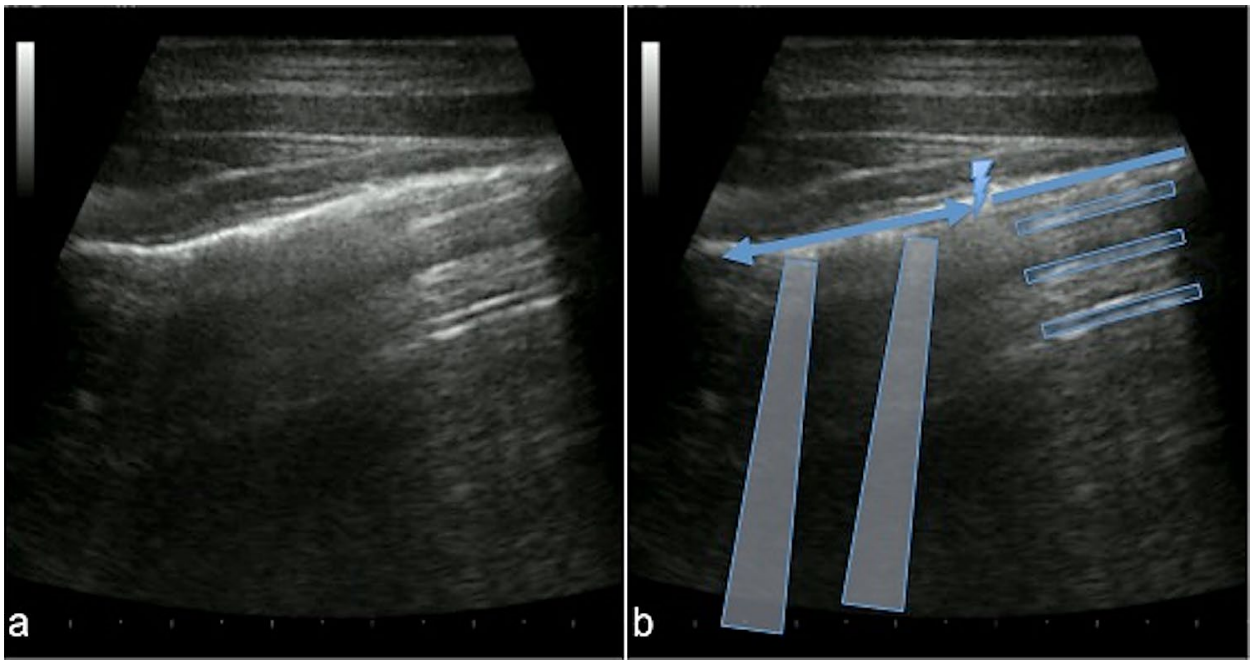

b) [14]. They are generated by distinct mechanisms: while reverberation due to acoustic impedance difference (soft tissue/metals; bile/cholesterol; soft tissue/gas) is the mechanism of generating comet tail artifacts, the B lines are caused by resonant vibration due to bubble tetrahedral complexes or their equivalents (Fig. 14c, d) [14, 37]. Using pneumothorax and interstitial syndrome as examples, the lung comets and $\mathrm{B}$-lines have distinct roles in disease diagnosis and the potential pitfalls if they are simply lumped together and called comet tail artifacts (Table 3) [44]. 
Fig. 13 Mirror image artifact (a), showing a similar tissuelike pattern in comparison with the liver (b blue area). Hyperechoic diaphragmatic line (b curved dotted line)
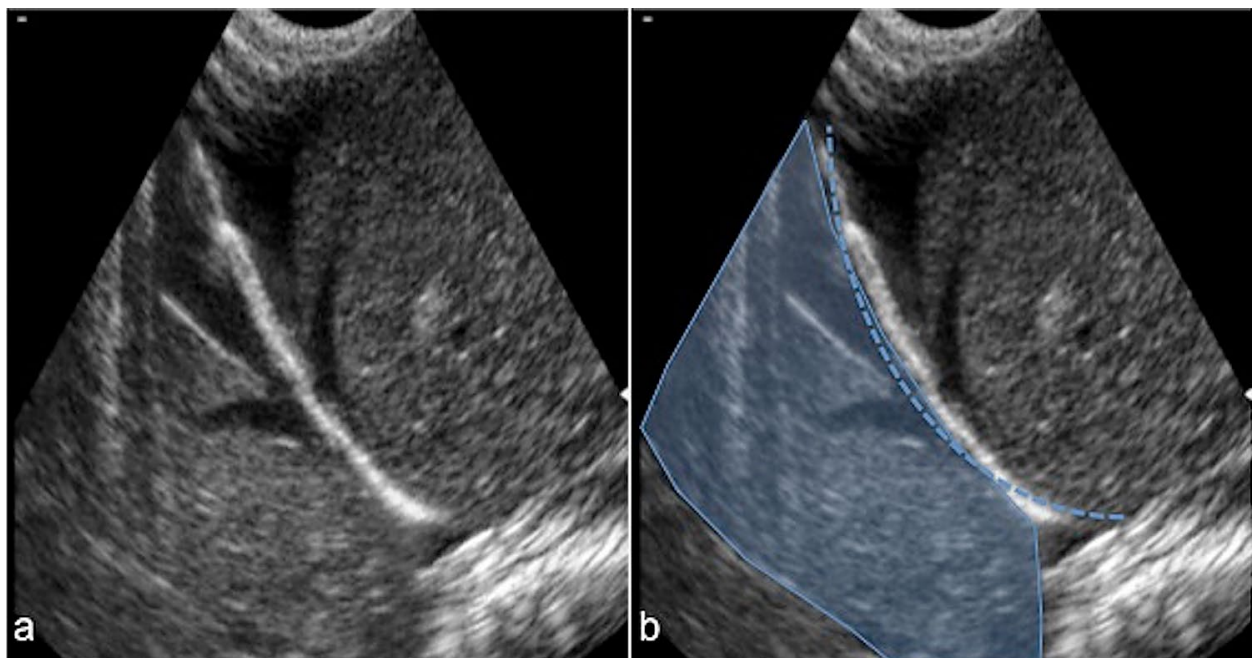

Fig. 14 Comet tail artifact (a-d). Chest ultrasound examination performed with a high frequency linear probe $(15-7 \mathrm{MHz})$ in healthy adult $(\mathbf{a}, \mathbf{b})$. Comet tail artifacts (a) with schematic representation (b). Small linear hyperechogenic artifacts come from the "pleural line" (b thickened line) and progress caudally for a few $\mathrm{mm}$ (b blue truncated cone). B lines (b blue vertical trapezoids) and A-lines (b blue box) are also represented. Gallbladder ultrasound performed with a low frequency convex probe $(5-10 \mathrm{MHz})(\mathbf{c}$, d). Comet tail artifacts (c) with schematic representation $(\mathbf{d})$ : comet tail artifacts are the result of cholesterol crystals deposited in Rokitansky-Aschoff sinuses (d blue truncated cone)

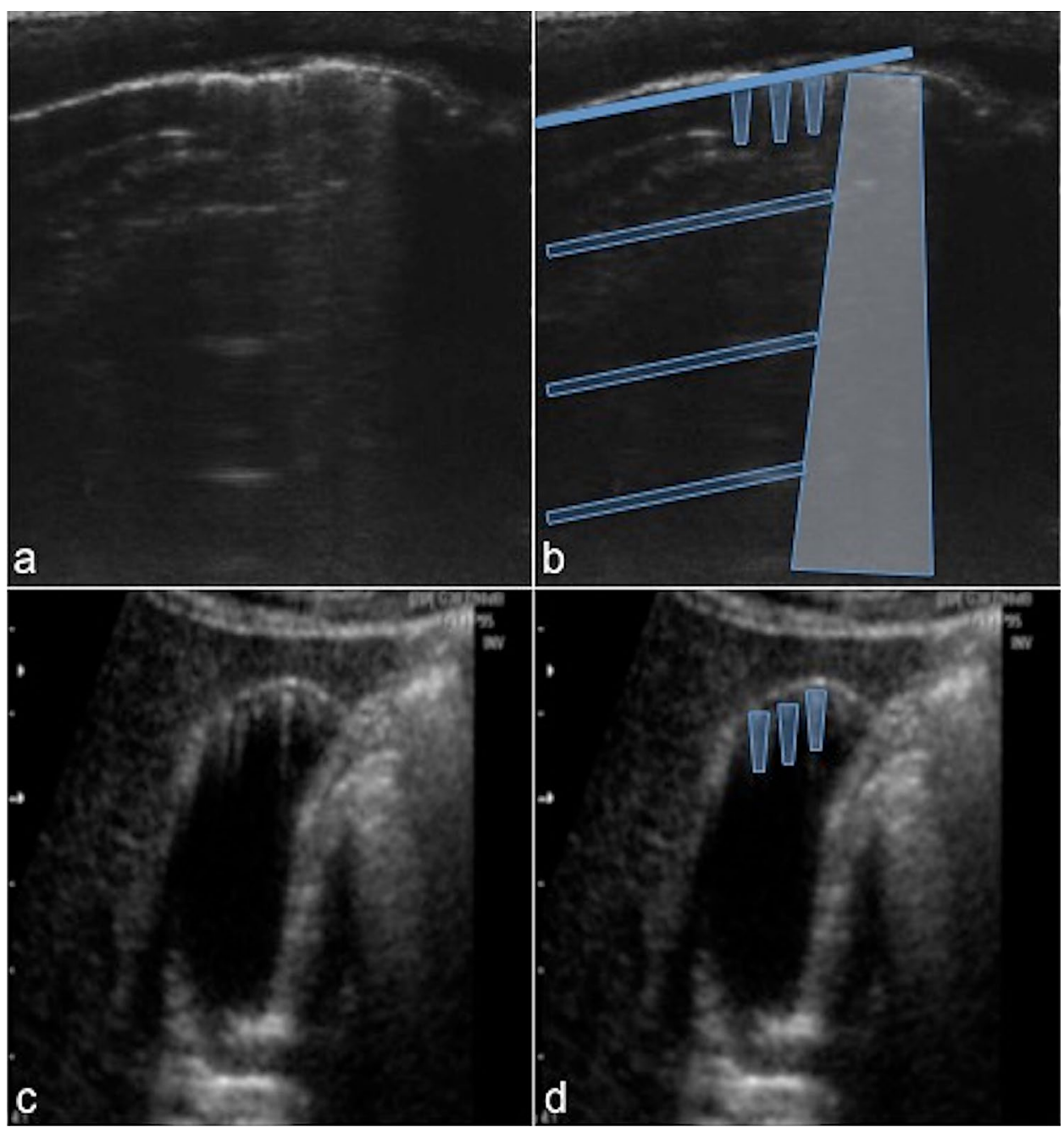

\section{Conclusion}

This brief review outlines the need to consider LUS exclusively into clinical and laboratory assessment according to validated protocols, as a synthesis of an already known acute clinical condition. For the emergency physician, bedside LUS is undoubtedly an important tool to 


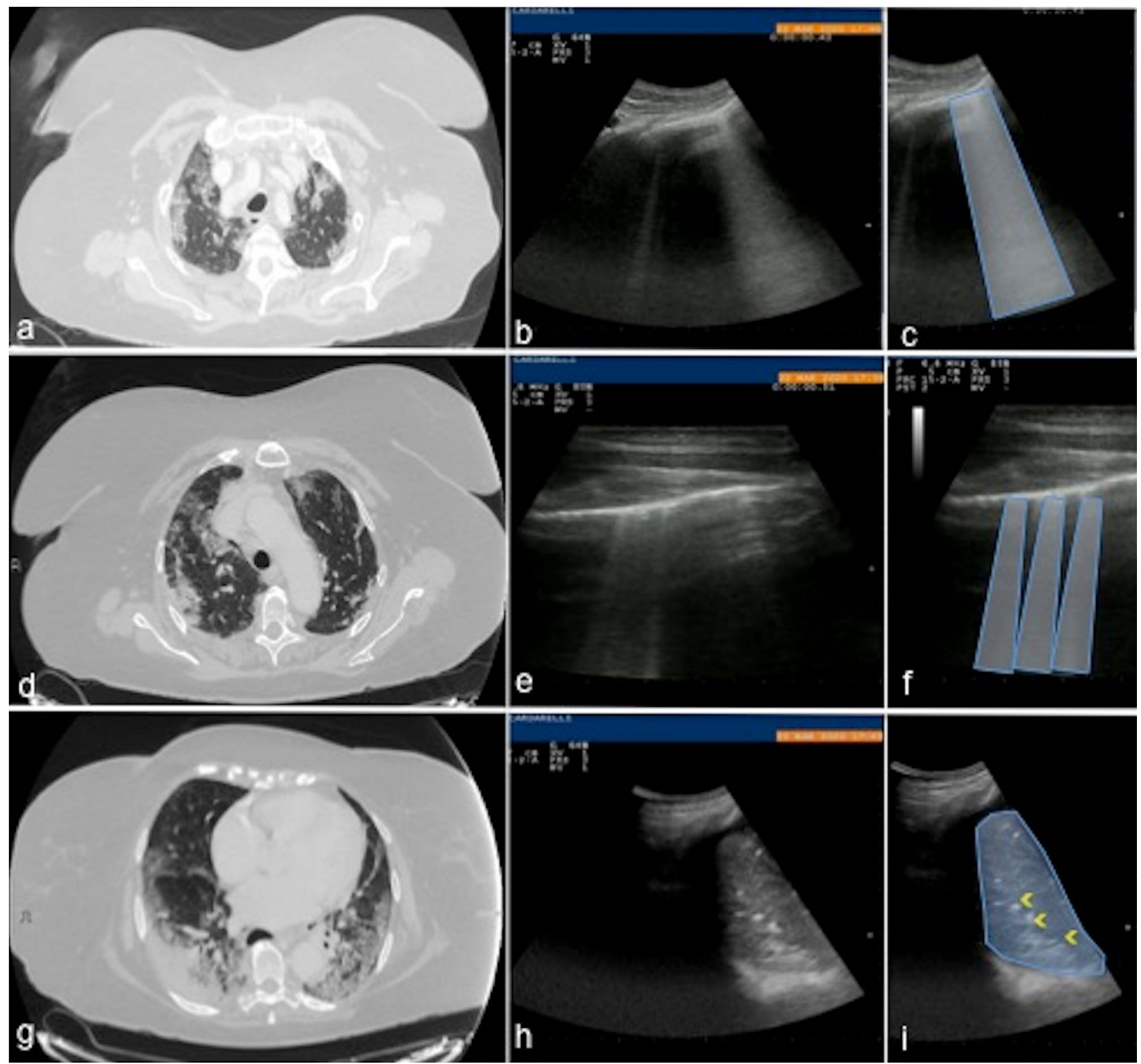

Fig. 15 Axial chest CT scan in lung window view $(\mathbf{a}, \mathbf{d}, \mathbf{g})$ at the pulmonary apices (a), upper lobes (d) and lower lobes (g) of a 65 yearsold female patient with fever shows: multiple, extensive areas of ground glass opacities in both lung (a, d), coexisting with consolidative opacities and "dark bronchogram sign" (g), consistent with COVID-19 rapid progression stage (positive for COVID-19 nasalpharyngeal swap RT-PCR). The corresponding ultrasound scans with low-frequency convex probe at right apex (b), linear high-frequency probe at right upper chest zone (e) and convex low-frequency probe at the right lower chest zone (h) show: vertical artifacts originate from the pleura line with a coalescence aspect similar to "white lung" pattern (blue trapezoid, c) at the right apex (b); multiple B lines (f blue trapezoids) at the right superior lobe (e); area of consolidation (i blue area) with air bronchogram (i yellow arrowheads) at the right base (h) narrow the differential diagnosis in the acutely dyspnoeic patient, who is too unstable to leave the department for further imaging exams. An ultrasound-based approach can save time in the assessment of dyspnoea, but it needs to be integrated with a standard clinical approach to optimize diagnostic accuracy. Given to its intuitive semeiotic, LUS is easy to perform in critical clinical contexts and can provide a wealth of information for the emergency physician, as well as to assess for fluid overload and whether a patient is a fluid tolerant for resuscitative measures. LUS permits also a dynamic study of patients with a significant impact on the clinical decision-making process of the critical patient [45]. According to our point of view, LUS is neither an anatomical ultrasound, but a clinical-interpretation of some artifacts, nor an alternative imaging exam to chest $\mathrm{X}$-ray or CT, but strongly integrated with them. At the 
same time, it appears evident that its use, also in new critical contexts, such as in the management of the COVID19 patient, can be part of this dualism, certainties, and uncertainties of the LUS, which can be overcome with the diagnostic integration with a X-ray at least or CT as starting point (Fig. 15). The described LUS intrinsic limits may affect data interpretation, especially when LUS is improperly used as a diagnostic screening test for generic lung diseases. Therefore, we recommend its use as a clinical and diagnostic complementary and monitoring tool.

\section{Compliance with ethical standards}

Conflict of interest The Authors declare that they have no conflict of interest.

Informed consent All procedures followed were in accordance with the ethical standards of the responsible committee on human experimentation (institutional and national) and with the Helsinki Declaration of 1975, and its late amendments. Additional informed consented was obtained from all patients for which identifying information is not included in this article.

Ethical Statement This article does not contain any studies with human or animal subjects performed by any of the Authors.

\section{References}

1. Henwood PC (2019) Imaging an outbreak-ultrasound in an Ebola treatment unit. N Engl J Med 4 381(1):6-9. https://doi. org/10.1056/nejmp1902830

2. Soldati G, Smargiassi A, Inchingolo R et al (2020) Proposal for international standardization of the use of lung ultrasound for COVID-19 patients; a simple, quantitative, reproducible method. J Ultrasound Med. https://doi.org/10.1002/jum.15285

3. Sofia S, Boccatonda A, Montanari M et al (2020) Thoracic ultrasound and SARS-COVID-19: a pictorial essay. J Ultrasound. https ://doi.org/10.1007/s40477-020-00458-7

4. Secco G, Delorenzo M, Zattera C, Giacomuzzi Moore B, Demitry L, Vezzoni G, Resta F, Barcella B, Cappa G, Perrone T, Salinaro F, Perlini S (2020) Lung ultrasound in COVID-19: a useful diagnostic tool. Emer Care J. https://doi.org/10.4081/ecj.2020.9017

5. Ticinesi A, Lauretani F, Nouvenne A et al (2016) Lung ultrasound and chest X-ray for detecting pneumonia in an acute geriatric ward. Medicine (Baltimore) 95(27):e4153. https://doi. org/10.1097/MD.0000000000004153

6. Maw AM, Hassanin A, Ho PM et al (2019) Diagnostic accuracy of point-of-care lung ultrasonography and chest radiography in adults with symptoms suggestive of acute decompensated heart failure: a systematic review and meta-analysis. JAMA Netw Open 1 2(3):e190703. https://doi.org/10.1001/jamanetworkopen 2019.0703

7. Touw HR, Parlevliet KL, Beerepoot M et al (2018) Lung ultrasound compared with chest X-ray in diagnosing postoperative pulmonary complications following cardiothoracic surgery: a prospective observational study. Anaesthesia 73(8):946-954. https:// doi.org/10.1111/anae.14243 (Epub 2018 Mar 12)
8. Lichtenstein DA (2014) Lung ultrasound in the critically ill. Ann Intensive Care 9 4(1):1. https://doi.org/10.1186/2110-5820-4-1

9. Deeb MA, Barbic S, Featherstone R et al (2014) Point-of-care ultrasonography for the diagnosis of acute cardiogenic pulmonary edema in patients presenting with acute dyspnea: a systematic review and meta-analysis. Acad Emerg Med 21(8):843-852. https ://doi.org/10.1111/acem.12435

10. Lichtenstein DA, Mezière GA (2008) Relevance of lung ultrasound in the diagnosis of acute respiratory failure: the BLUE protocol. Chest 134(1):117-125. https://doi.org/10.1378/chest $.07-2800$

11. Wongwaisayawan S, Ruedeekorn S, Sawatmongkorngul S et al (2016) Emergency thoracic US: the essentials. Radiographics 36(3):640-659. https://doi.org/10.1148/rg.2016150064

12. Koh DM, Burke S, Davies N et al (2002) Transthoracic US of the chest: clinical uses and applications. Radiographics 22:E1

13. Mojioli F, Bouhemad B, Mongodi S et al (2019) Lung ultrasound for critically Ill patients. Am J Respir Crit Care Med 15 199(6):701-714. https://doi.org/10.1164/rccm.201802-0236ci

14. Rea G, Sperandeo M, Di Serafino M et al (2019) Neonatal and pediatric thoracic ultrasonography. J Ultrasound 22(2):121-130. https://doi.org/10.1007/s40477-019-00357-6

15. Lichtenstein DA, Menu Y (1995) A bedside ultrasound sign ruling out pneumothorax in the critically ill. Lung sliding. Chest 108:1345-1348

16. Husain LF, Hagopian L, Wayman D et al (2012) Sonographic diagnosis of pneumothorax. J Emerg Trauma Shock 5(1):76-81. https://doi.org/10.4103/0974-2700.9311

17. Lichtenstein DA, Lascols N, Prin S et al (2003) The "lung pulse": an early ultrasound sign of complete atelectasis. Intensive Care Med 29(12):2187-2192. https://doi.org/10.1007/s00134-0031930-9 (Epub 2003 Oct 14)

18. Lubna FH, Laura H, Derek W et al (2012) Sonographic diagnosis of pneumothorax. J Emerg Trauma Shock 5(1):76-81. https://doi. org/10.4103/0974-2700.93116

19. Lichtenstein D, Mezière G, Seitz J (2009) The dynamic air bronchogram A lung ultrasound sign of alveolar consolidation ruling out atelectasis. Chest 135(6):1421-1425. https://doi.org/10.1378/chest .08-2281 (Epub 2009 Feb 18)

20. Neto Francisco MJ, Junior Rahal A, Vieira FA et al (2016) Advances in lung ultrasound. Einstein (Sao Paulo) 14(3):443-448. https://doi.org/10.1590/S1679-45082016MD3557

21. Lichtenstein DA (2015) BLUE-protocol and FALLS-protocol: two applications of lung ultrasound in the critically ill. Chest 147(6):1659-1670. https://doi.org/10.1378/chest.14-1313

22. Hernandez C, Shuler K, Hannan H et al (2008) C.A.U.S.E.: cardiac arrest ultra-sound exam-a better approach to managing patients in primary non-arrhythmogenic cardiac arrest. Resuscitation 76(2):198-206 (Epub 2007 Sep 5)

23. Cavaliere F, Biasucci D, Costa R et al (2011) Chest ultrasounds to guide manual reexpansion of a postoperative pulmonary atelectasis: case report. Minerva Anestesiol 77(7):750-753

24. Tsubo T, Yatsu Y, Tanabe T et al (2004) Evaluation of density area in dorsal lung region during prone position using transesophageal echocardiography. Crit Care Med 32(1):83-87

25. Biasucci DG, Ricci Z, Conti G et al (2014) Sonographic dynamic assessment of lung injury in a child with hypoplastic left heart syndrome undergoing extracorporeal membrane oxygenation. Pediatr Pulmonol 49(12):E147-E150. https://doi.org/10.1002/ ppul.23059

26. Lichtenstein DA (2019) Current misconceptions in lung ultrasound: a short guide for experts. Chest 156(1):21-25. https://doi. org/10.1016/j.chest.2019.02.332

27. Sperandeo M, Tinti MG, Rea G et al (2017) Chest ultrasound versus chest $\mathrm{X}$-rays for detecting pneumonia in children: why 
compare them each other if together can improve the diagnosis? Eur J Radiol 93:291-292. https://doi.org/10.1016/j.ejrad .2017.05.038

28. Gargani L, Volpicelli G (2014) How I do it: lung ultrasound. Cardiovasc Ultrasound 12:25. https://doi.org/10.1186/1476-7120-12-25

29. Rea G, Trovato MG (2015) A farewell to B lines: ageing and disappearance of ultrasound artifacts as a diagnostic tool. Respiration 90(6):522. https://doi.org/10.1159/000441010 (Epub 2015 Oct 7)

30. Mozzini C, Fratta Psini AM, Garbin U et al (2016) Lung ultrasound in internal medicine: training and clinical practice. Crit Ultrasound J 1:10. https://doi.org/10.1186/s13089-016-0048-6

31. Frongillo E, Gaudioso G, Feragalli B (2020) Ultrasound and interstitial lung disease: use and limitations. Radiol Med 125(1):66-67. https://doi.org/10.1007/s11547-019-01084-y

32. Brusasco C, Santori G, Bruzzo E et al (2019) Quantitative lung ultrasonography: a putative new algorithm for automatic detection and quantification of B-lines. Crit Care 28 23(1):288. https://doi. org/10.1186/s13054-019-2569-4

33. Price S, Platz E, Cullen L et al (2017) Expert consensus document: echocardiography and lung ultrasonography for the assessment and management of acute heart failure. Nat Rev Cardiol 14(7):427-440. https://doi.org/10.1038/nrcardio.2017.56 (Epub 2017 Apr 27)

34. Foti T, D'amato M, Rea G (2015) Insufficient proof of concept for using ultrasound to guide fluid resuscitation. Anesthesiology 123(3):718. https://doi.org/10.1097/ALN.0000000000000787

35. Del Colle A, Carpagnano GE, Feragalli B et al (2019) Transthoracic ultrasound sign in severe asthmatic patients: a lack of "gliding sign" mimic pneumothorax. BJR Case Rep 15 5(4):20190030. https://doi.org/10.1259/bjrcr.20190030

36. Frongillo E, Rea G, Tinti MG et al (2017) Limitations of focused assessment with sonography in trauma (FAST) protocols in transthoracic US. Radiology 285(2):693-694. https://doi.org/10.1148/ radiol.2017171692

37. D'Amato M, Rea G, Carnevale V et al (2017) Assessment of thoracic ultrasound in complementary diagnosis and in follow up of community-acquired pneumonia (cap). BMC Med Imaging 31 17(1):52. https://doi.org/10.1186/s12880-017-0225-5

38. Tinti MG, Rea G, Mirijello A et al (2017) Is there any role for thoracic ultrasound for interstitial lung disease underlying rheumatologic conditions? Comment. Intern Emerg Med 12(6):903-904. https://doi.org/10.1007/s11739-017-1663-3
39. Gutierrez M, Tardella M, Rodriguez L et al (2019) Ultrasound as a potential tool for the assessment of interstitial lung disease in rheumatic patients. Where are we now? Radiol Med 124(10):989999. https://doi.org/10.1007/s11547-019-01053-5

40. Lichtenstein DA, Lascols N, Mezière G et al (2004) Ultrasound diagnosis of alveolar consolidation in the critically ill. Intensive Care Med 30:276-281. https://doi.org/10.1007/s0013 4-003-2075-6

41. Laursen CB, Graumann O, Davidsen JR et al (2014) Pitfall in lung ultrasound: 'pseudo B-line' seen in both hydropneumothorax and in a cup of coffee. BMJ Case Rep 28:2014. https://doi. org/10.1136/bcr-2013-201341

42. Cipriani C, D'agostino F, Rea G (2020) Diagnosis of pneumothorax in major trauma: fast or accuracy? J Ultrasound. https://doi. org/10.1007/s40477-020-00444-z

43. Blanco P, Volpicelli G (2016) Common pitfalls in point-of-care ultrasound: a practical guide for emergency and critical care physicians. Crit Ultrasound J 8:15. https://doi.org/10.1186/s1308 9-016-0052-x

44. Yue Lee FC, Jenssen C, Dietrich CF (2018) A common misunderstanding in lung ultrasound: the comet tail artefact. Med Ultrason 30 20(3):379-384. https://doi.org/10.11152/mu-1573

45. Xirouchaki N, Kondili E, Prinianakis G et al (2014) Impact of lung ultrasound on clinical decision making in critically ill patients. Intensive Care Med 40:57-65. https://doi.org/10.1007/s0013 4-013-3133-3

46. Elmahalawya II, Dohab NM, Ebeidc OM, Abdel-Hadyd MA, Saiedb O (2017) Role of thoracic ultrasound in diagnosis of pulmonary and pleuraldiseases in critically ill patients. Egypt J Chest Dis Tuberc 66(2):261-266

47. Karimi E (2019) Comparing sensitivity of ultrasonography and plain chest radiography in detection of pneumonia; a diagnostic value study. Arch Acad Emerg Med. 7(1):e8

Publisher's Note Springer Nature remains neutral with regard to jurisdictional claims in published maps and institutional affiliations. 